COMMUNICATIONS

\title{
VASCULAR ANATOMY OF THE CHOROID IN RELATION TO SELECTIVE LOCALIZATION OF OCULAR DISEASE*
}

BY

\author{
KENNETH C. WYBAR \\ Department of Pathology, Institute of Ophthalmology, London
}

THE choroidal coat of the eye consists of a dense continuous network of vessels and yet it is a striking feature of choroidal disease that the lesions often remain remarkably circumscribed. It is the purpose of this paper to review the problem of selective localization of pathological changes within the choroid in relation to its vascular anatomy, with particular reference to certain findings (Wybar, 1954) recently made during a study of Neoprene latex casts of the choroidal vessels of the human eye prepared by the intravascular injection technique of Ashton (1952). The cohesive and elastic properties of Neoprene permit dissection of individual vessels or capillary areas from the main vascular mass, and these can then be studied in detail using a wide-field stereoscopic microscope with direct illumination from a high-power low-voltage filament lamp. This technique represents a distinct advance on former dye injection methods, particularly in such a densely vascular tissue as the choroid.

\section{Previous Theories in the Literature}

\section{Theory of Division into Sectors}

The experimental investigations of Wagenmann (1890) on the rabbit gave rise to the belief that the short posterior ciliary arteries are functional endarteries and that the vessels within the choroid have a strictly segmental distribution. Wagenmann found that, when he sectioned a few short posterior ciliary arteries behind the globe in the living animal, a localized area of choroidal atrophy occurred, which corresponded in situation to the field of distribution of the affected vessels and was sharply demarcated from the surrounding healthy choroid. There was a tendency for some of the vessels in the affected zone to re-open within a few days, but in many of the animals a small sector of permanent vascular obliteration remained. The detailed study of the choroidal circulation by Leber (1903) contributed to this concept by the emphasis which he laid on the absence of anastomoses between the various branches of the short posterior ciliary arteries except in the immediate vicinity of the optic disc and in the extreme periphery of the choroid.

Certain clinical observations have been advanced to substantiate Wagenmann's findings of a segmental pattern of the choroidal vascular supply. A wedge-shaped area of choroidal degeneration was described by Coats (1907) in a case of posterior scleritis due, he presumed, to occlusion of a short posterior ciliary artery in the sclera when this tissue was inflamed. Hepburn 
(1912) also believed that the blockage of a short posterior ciliary artery might produce a wedge-shaped defect in the choroid, although he based this conclusion entirely on an interpretation of the changes in the visual fields in certain cases showing small deep foci of choroiditis. In such cases he found a sector-shaped scotoma extending from the focus of choroiditis to the periphery of the visual field, and he considered that this indicated a circulatory failure within the whole underlying sector of choroid due to the involvement of a short posterior ciliary artery in the inflammatory focus.

\section{Theory of Division into Zones}

In addition to the concept that the choroid is divided into discrete radially arranged sectors, each of which extends from the peripapillary region to the peripheral limit of the choroid, it has been postulated that there are several specialized and distinct zones within the choroid, which include the equator, the periphery, the region underlying the macula, and the peripapillary zone. The anatomical and clinical factors which have led to this view are reviewed below.

Equatorial Choroid.-This zone was considered by Leber (1903) to be a peculiar region of the choroid in that it represented the meeting place of the terminal twigs of the short posterior ciliary arteries running forwards, and of the recurrent choroidal arteries from the anterior part of the uveal tract running backwards; Leber regarded these two systems as fairly separate entities, despite their association at the equator through an intervening capillary anastomosis. This view of an ineffective anastomosis at the equator was also held by Gonin (1903) and Nettleship (1903), who considered that the resulting poverty in blood supply in that region rendered it particularly vulnerable to pathological conditions affecting the choroidal vascular bed. This conclusion was derived from a study of the changes in the visual fields in cases of retinitis pigmentosa, and was based on the hypothesis that the retinal degeneration resulted from a defect in the underlying choroidal circulation. In this way the ring scotoma, the characteristically early visual field change in retinitis pigmentosa, was interpreted as indicating a preferential disturbance of the equatorial part of the choroid.

Peripheral Choroid.-The zone in the extreme periphery of the fundus has been considered to be a specialized area; indeed, this is the logical outcome of an acceptance of the view that the anterior vascular supply is partially isolated from the rest of the choroid by the imperfect anastomosis between the two vascular systems at the equator. Evidence suggesting a peculiarity in the structure of this region of the choroid was advanced by Hancock (1908), who pointed out that the function of the extreme periphery of the retina may be retained in certain cases of total occlusion of the central retinal artery. He regarded this as an indication of a specialization of the chorio-capillaris in the extreme periphery, so that it is capable of nourishing the whole thickness of the retina, in contrast to the chorio-capillaris elsewhere, which can maintain the viability of the outer retinal layers only. The fact that transudate 
can filter through the whole thickness of the peripheral part of the retina has led to the suggestion that the anterior part of the choroid may contribute to the formation of aqueous humour (Duke-Elder, 1948); and this may be a further indication of a peculiarity of that part of the uvea.

Sub-Macular Choroid.-This area has been considered to be peculiar in two respects: first, in that its capillaries are more densely packed with a corresponding reduction in the inter-capillary spaces (Leber, 1903; Lauber, 1931), and second, in that it is more vascular because of a greater aggregation of vessels within its area (Wolfrum, 1908; Salzmann, 1912; Lauber, 1936; Mörike, 1949). The concept of a specialized structure of the choroidal vascular bed at the macula is of particular interest in view of the frequency with which certain pathological processes affecting the uveal tract remain confined to the posterior pole of the eye. For example, the choroidal haemorrhage which impairs the function of the retina in certain senile eyes and in advanced myopia occurs characteristically in this region, and cystoid degenerative changes of the retina, which follow degeneration of the underlying chorio-capillaris, is characteristically limited to the macula; indeed it has been shown pathologically (Ashton, 1953) that extensive atrophy of the chorio-capillaris in the macular and paramacular regions may occur in central senile areolar choroidal sclerosis without any similar involvement of the rest of the choroid. The localization of this disease to the central part of the fundus prompted Nettleship (1903) to put forward the hypothesis that it is based on a narrowing of the lumen of the short posterior ciliary arteries supplying the choroid at the posterior pole.

Peripapillary Choroid.-The area surrounding the optic disc has also been considered to be a specialized zone (Duke-Elder, 1940). A continuous arterial network in this region, composed of arterioles which do not participate in the vascular supply of the rest of the choroid, was described by Marín-Amat (1952), who suggested that certain forms of contusion of the eye may rupture these fine vessels by suddenly stretching and twisting the globe. Involvement of this vascular ring would thus be followed by the development of a peripapillary choroidal atrophy (Bartolozzi, 1952).

\section{Objections to these Theories}

All this evidence, apparently pointing to a rigid segmentation of the posterior ciliary vessels and to a regional characterization of certain areas of the choroid and its capillary network, forms a particularly attractive thesis in considering the selective localization of choroidal disease, but there are findings which are opposed to such a concept:

(1) There is doubt regarding the validity of the hypothesis that the arteries in the choroid maintain a rigid independence of one another, and that they, therefore, have the characteristics of end-arteries.

Each short posterior ciliary artery, as it perforates the sclera in the posterior part of the globe, breaks up into many branches which run forward in narrow 

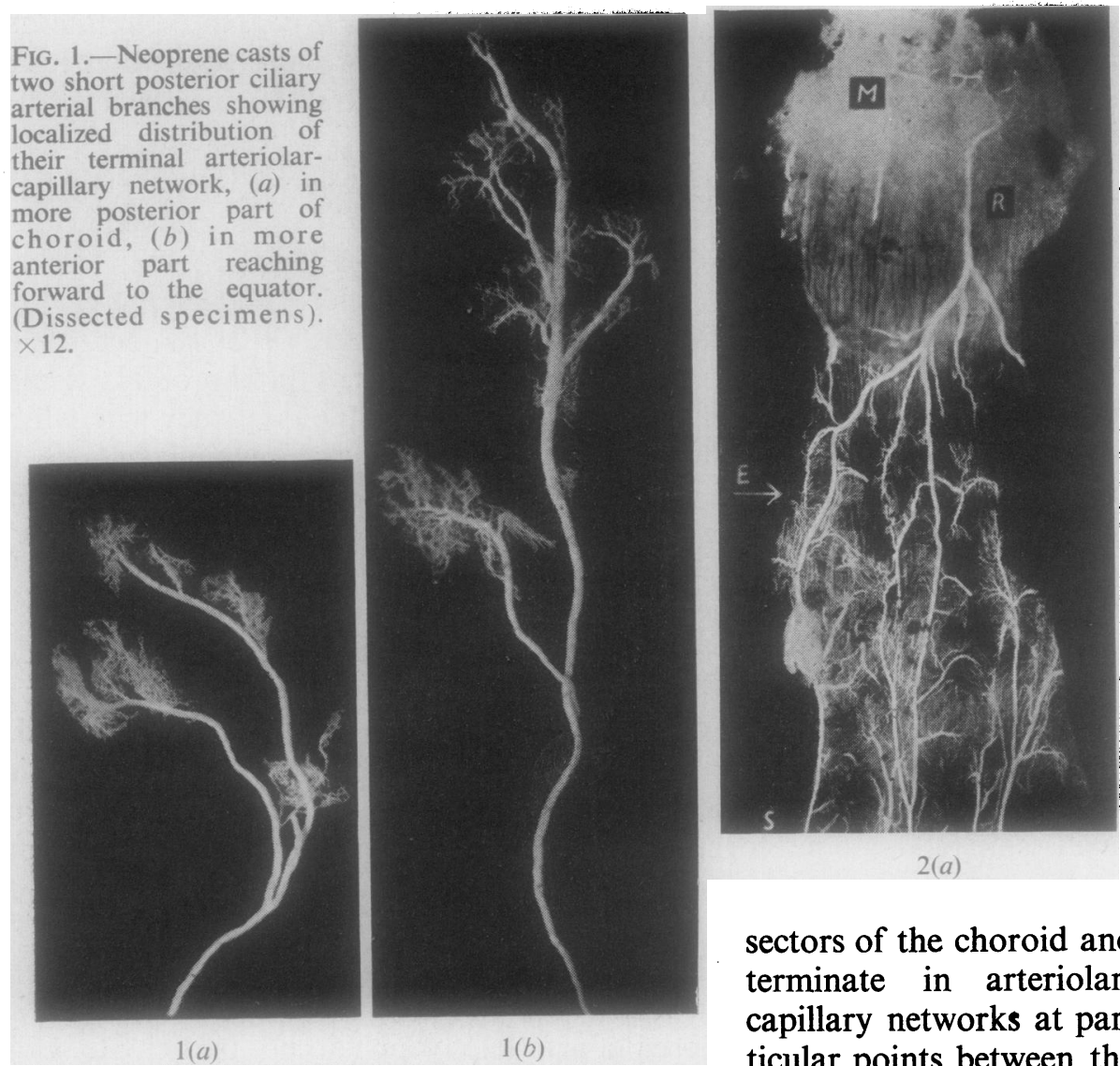

sectors of the choroid and terminate in arteriolarcapillary networks at particular points between the peripapillary region and the equator of the eye (Figs $1 a$ and $1 b$ ). These arteriolar-capillary networks, however, do not form isolated units because they are linked with each other through the chorio-capillaris, which lies as a continuous capillary layer on the inner surface of the choroid. Furthermore, many of the short posterior ciliary arteries which run forward to the equator come into contact there with the recurrent choroidal arteries arising from three sources: the major arterial circle of the iris (Fig. 2a), the long posterior ciliary arteries (Fig. $2 b$,opposite), and the anterior ciliary arteries (Fig. 2c, opposite); and, although this anastomosis may be of a capillary nature (Fig. 3, opposite), it has been shown (Wybar, 1954) that it is not uncommon for the opposing vessels to be directly continuous with one another (Figs $4 a$ and $4 b$, overleaf). The anterior part of the uveal circulation and the close anastomosis between the posterior and recurrent choroidal circulations at the equator, therefore, provide another pathway through which individual arteriolar-capillary networks may be linked to one another. 


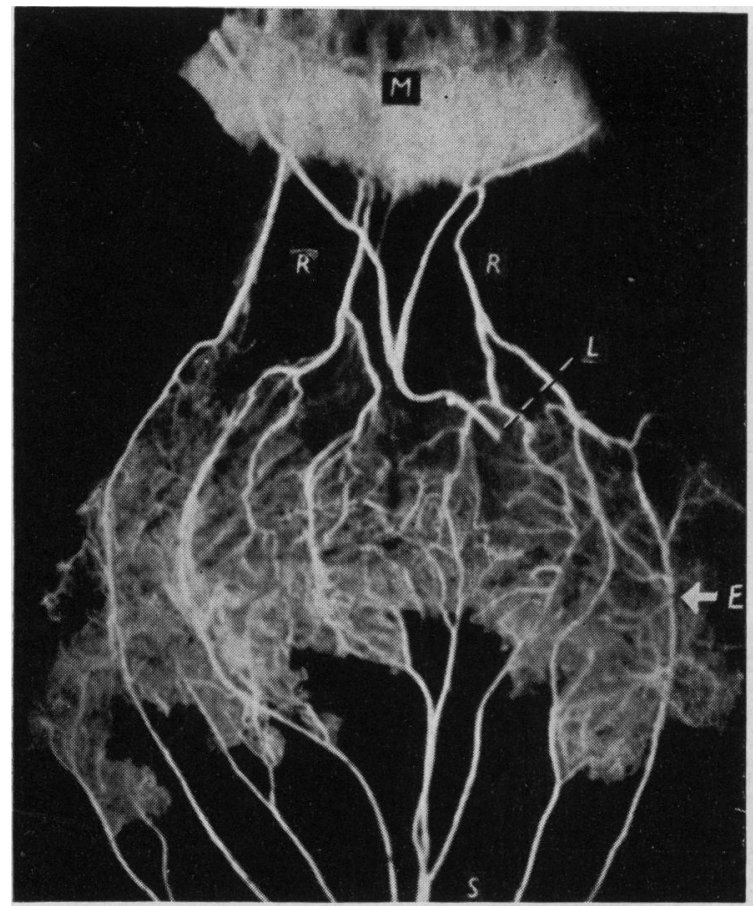

$2(b)$

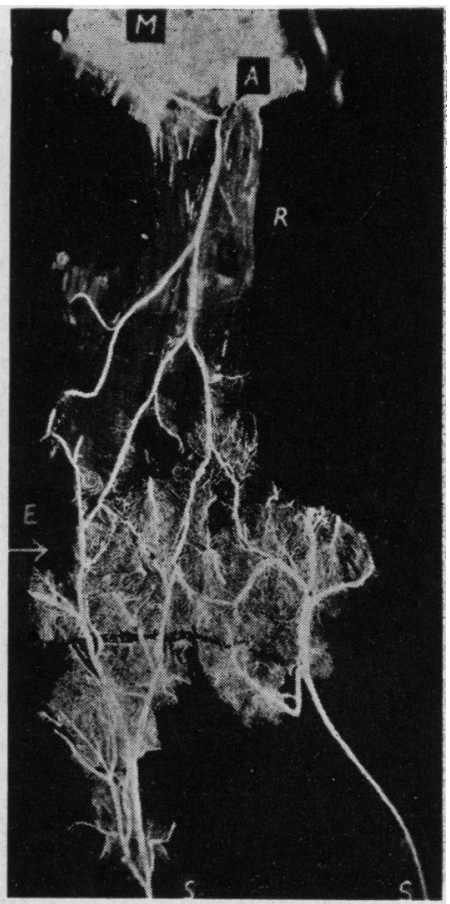

$2(c)$

FIG. 2.-Neoprene casts of three strips of uvea showing recurrent choroidal arteries (R) arising:

(a) from the major arterial circle of the iris (M);

(b) from a long posterior ciliary artery (L);

(c) from an anterior ciliary artery (A).

The terminal parts of several short posterior ciliary arterial branches (S) are seen below. Note direct continuity of the two arterial systems at equator (E), although in places there is an intervening capillary network. (Dissected specimens). $(a) \times 8 ;(b) \times 10 ;(c) \times 8$. (Wybar, 1954).

An attempt has been made to study this aspect of the choroidal circulation by injecting the choroid with Neoprene latex or with Indian ink through the ophthalmic artery after occlusion of a single short posterior ciliary artery in the orbit. In four eyes, two injected with Neoprene

Fig. 3.-Neoprene cast of terminal parts of recurrent choroidal artery $(R)$, and of short posterior ciliary arterial branch (S), showing intervening capillary network at equator (E). (Dissected specimen). $\quad \times 19$. (Wybar, 1954).

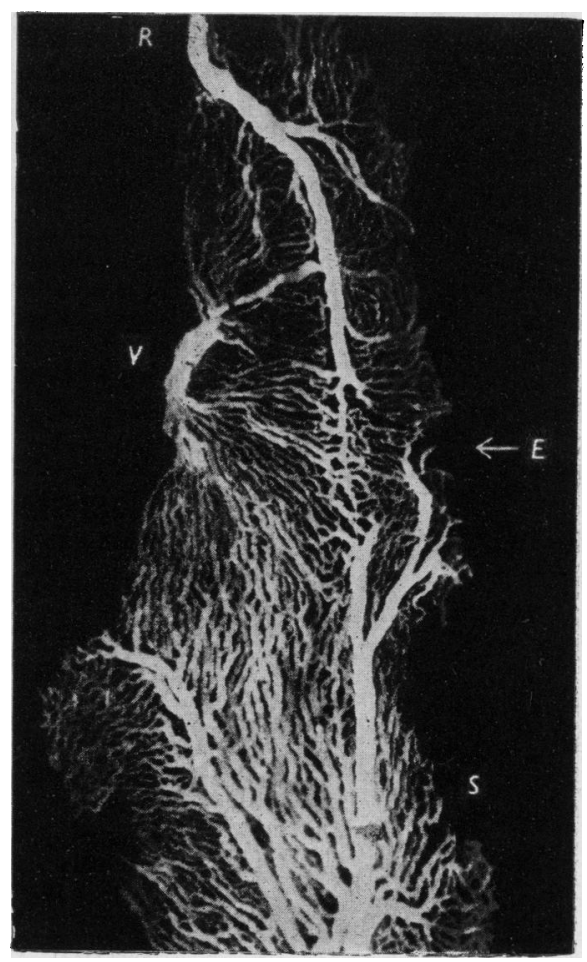




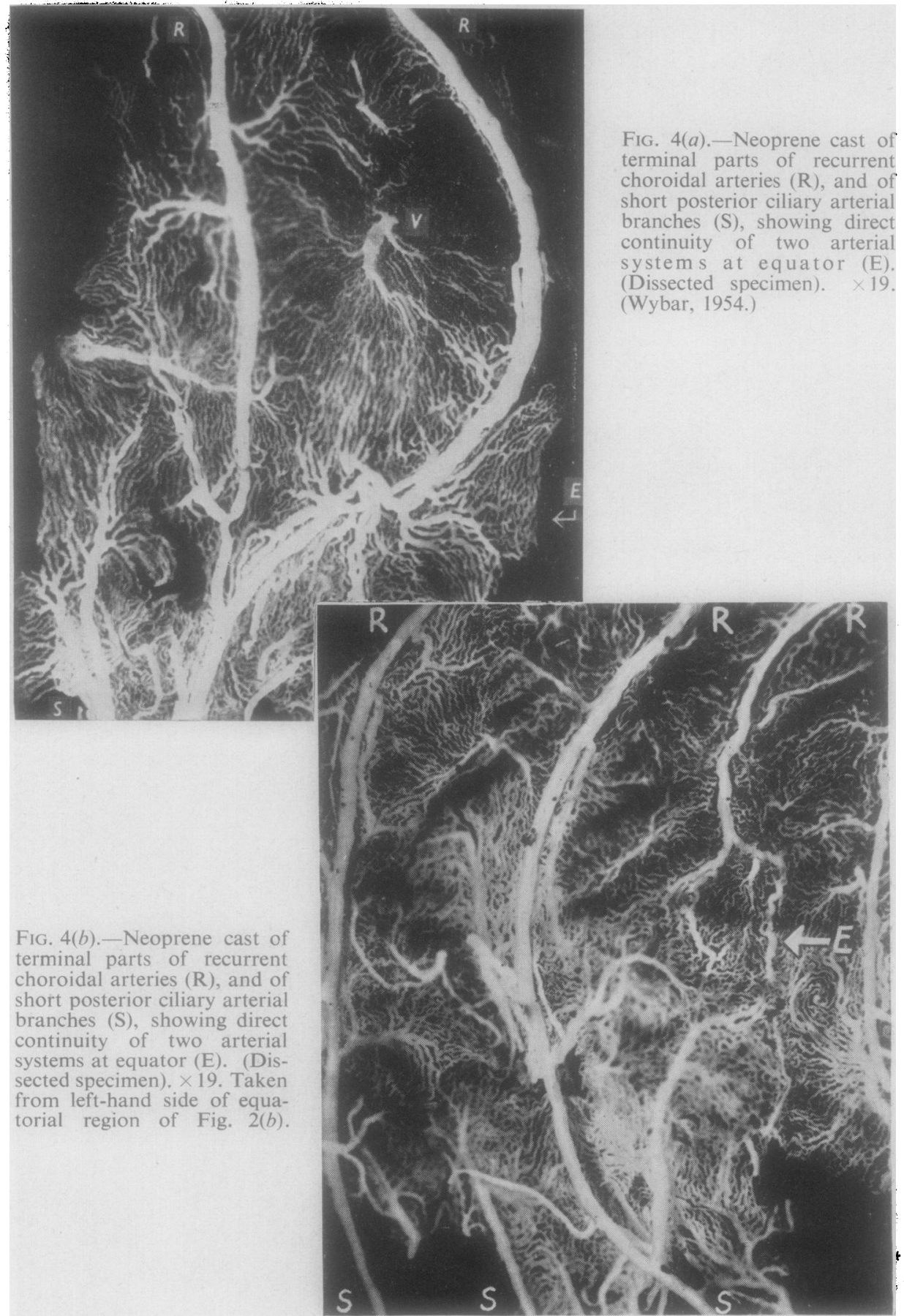




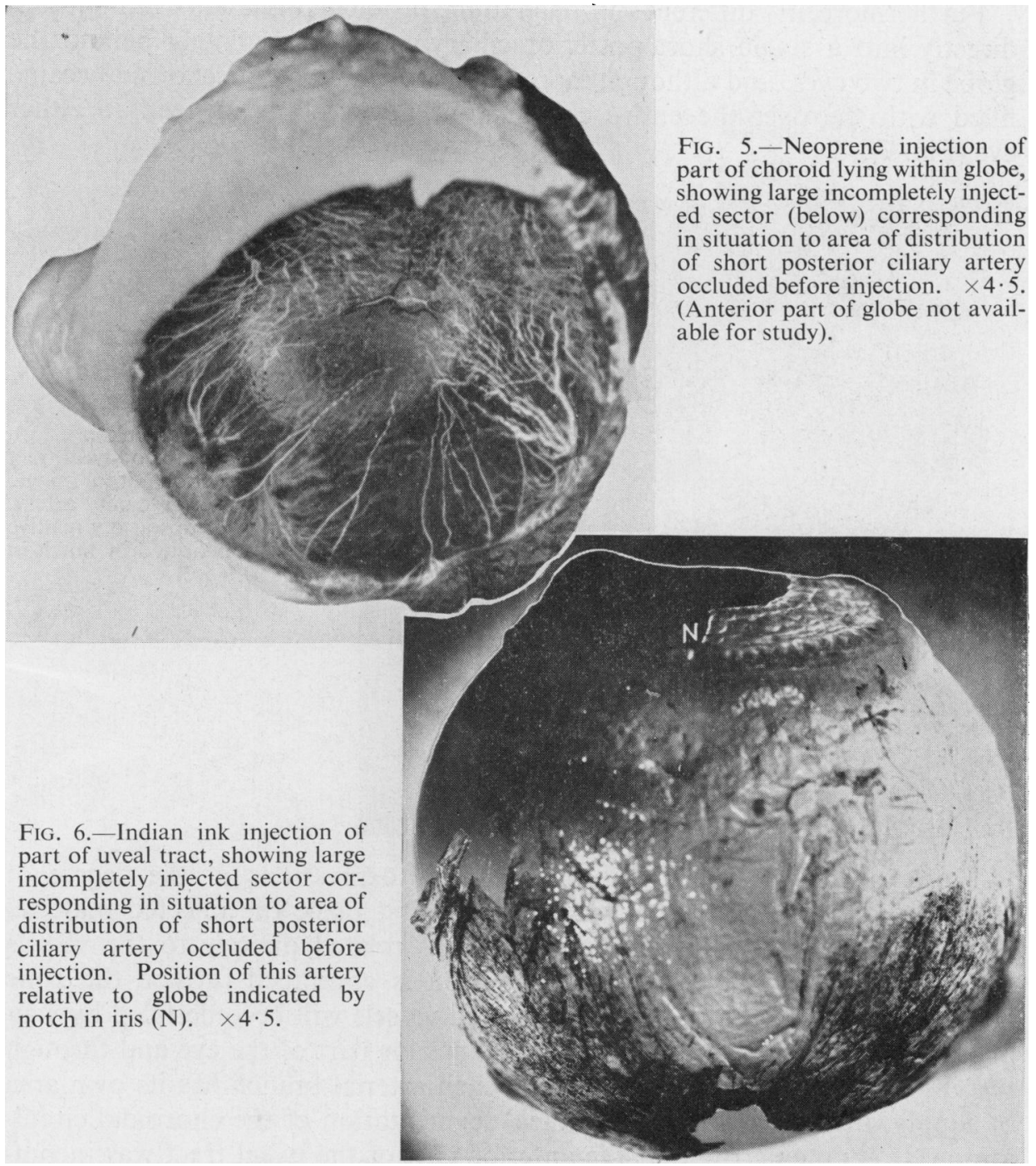

and two with Indian ink, the choroid was completely filled despite the ligation of the vessel; this would be expected, in view of the ease with which the injected material may pass from the posterior ciliary arteries through the equator to the anterior part of the uveal tract, where it is redistributed by the recurrent choroidal arteries to any sector of the choroid which has been inadequately filled from its own posterior ciliary vessels or from the neighbouring choriocapillaris. The results of this study were not consistent, however, because in three other eyes, two injected with Neoprene and one with Indian ink, a partial sectorial filling defect occurred in the choroid, corresponding in situation to the blocked short posterior ciliary artery (Figs 5 and 6). 
Furthermore, in a different approach to the problem, Indian ink was injected directly into a single short posterior ciliary artery immediately behind the globe in two eyes, and although more than two-thirds of the choroid became filled with the dye, the entire choroid did not become injected in either eye (Fig. 7).

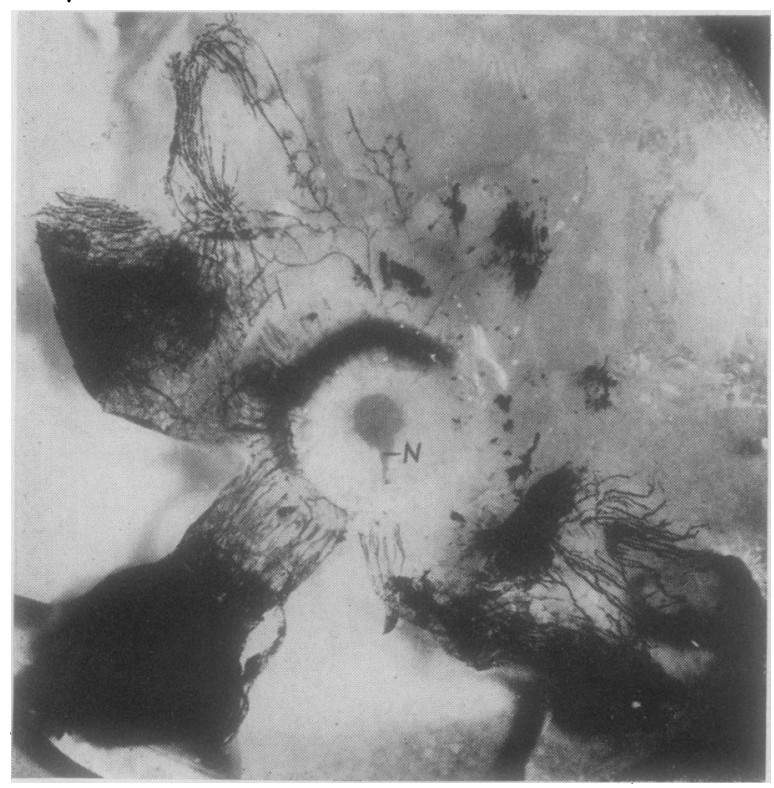

FIG. 7.-Indian ink injection of extensive area of uveal tract after injection into single short posterior ciliary artery. Position of this artery relative to globe indicated by notch in iris $(\mathbf{N}) . \quad \times 3$.

These inconsistencies are probably purely expressions of the degree of success of the injection technique. In the first place the injected material passes into the arteriolar-capillary networks related directly to the vessels which are being filled, but, if the injection is continued further, the zone dependent on the occluded or non-injected vessels will become filled by way of the alternative pathways through the anterior part of the eye and through the chorio-capillaris, so that, although each arterial branch has its own area of supply, there is no rigid anatomical segmentation of the choroidal circulation. It is noteworthy that the anterior part of the uveal tract was incompletely injected in the eyes which showed a sectorial filling defect (Fig. 6). It would be expected, therefore, on anatomical grounds, that in the event of a circulatory failure within a choroidal sector following occlusion of a short posterior ciliary artery, a restoration of circulation could occur from the other ciliary vessels.

This doubt regarding the rigid segmentation of the choroidal vessels was expressed by Nicholls (1938), who did not confirm the experimental findings of Wagenmann (1890). He found no structural changes within the choroid of the rabbit after occlusion of the short posterior ciliary arteries, unless at least two-thirds of the arteries were affected, in which case a phthisical condition of the eye was more likely to develop than a sectorial area of choroidal atrophy. It would also appear 
that the clinical cases which have been cited to illustrate the existence of choroidal segmentation are based on hypotheses which may not be valid. In the case described by Coats (1907), it is more likely that the wedge-shaped area of choroidal atrophy, found in association with a patch of posterior scleritis, was due to involvement of the choroidal sector in the scleral inflammation with consequent obliteration of the vessels and capillaries, rather than to indirect circulatory failure in the sector from blockage of a short posterior ciliary artery within the sclera. An area of choroid will not become atrophic unless its chorio-capillaris is affected, so that the pathogenesis suggested by Coats is valid only if the thrombotic process extends into the chorio-capillaris of the affected sector.

In the case described by Hepburn (1912), the sector-shaped scotoma extending to the periphery of the visual field, following a circumscribed patch of deep choroiditis, may be explained solely on the basis of involvement of the overlying retina in the inflammatory process, without postulating any circulatory failure within the whole sector of the choroid corresponding in shape to the visual field defect. If the choroidal inflammation involves only the outer layers of the overlying retina, the defect in the visual field is a localized scotoma corresponding in size and position to the focus of choroiditis, but if the inner retinal layers are also involved, the function of a whole sector of retina is affected, with the production of a scotoma extending to the periphery of the visual field. Further evidence that this is the probable interpretation of such cases was brought forward by Wolff and Penman (1950), who demonstrated that a narrow functional zone may remain between the localized and sectorial field defects. This would not be so if the field loss were due to a circulatory failure in a complete choroidal sector, but it can be explained on the basis of an involvement of the retinal nerve fibre layer.

(2) There is doubt regarding the validity of the hypothesis that the equator is a specialized area of the choroid in that it represents a zone of ineffective anastomoses between the posterior and recurrent choroidal circulations.

It has been shown above that many of the vessels of the two opposing circulations are in direct continuity with one another at the equator, and that there is no evidence of a defective circulation in this region (Figs $2 a, 2 b$, $2 c$, and 4). The concept of an ineffective anastomosis at the equator, although partly based on the anatomical studies of Leber (1903), was derived primarily from an interpretation of the visual field changes in retinitis pigmentosa, with the basic supposition that the retinal changes are secondary to a choroidal degeneration (Gonin, 1903; Nettleship, 1903). It is now known, however, that, although patches of choroidal angiosclerosis may occur in retinitis pigmentosa (Greeves, 1912), they are not an essential part of the pathological picture (Cogan, 1950), and may indeed be completely absent (Collins, 1919; Verhoeff, 1931). Experimental evidence of a hereditary pigmentary degeneration of the retina in rats (Bourne, Campbell, and Tansley, 1938) and in dogs (Parry, 1953), a condition which appears to be akin to retinitis pigmentosa, also indicates a primary retinal disturbance without any related choroidal lesions. There is, therefore, no sound basis for drawing conclusions regarding the state of the choroidal circulation from a study of the retina in retinitis pigmentosa; and the finding of an effective anastomosis at the equator does not conflict with modern views on the pathogenesis of the disease. 
In this connexion it should be noted that the view put forward by Hepburn (1910), that the choroid is a series of terminal vascular systems, which, in disease, may be separately affected without necessarily involving neighbouring vessels, was also based to a certain extent on an interpretation of the visual field changes in retinitis pigmentosa in the belief that the disease is a primary choroidal affection. The choroid was divided by Hepburn into three main zones: macular, mid-peripheral, and extreme peripheral, but it should be remembered that these terms were used to denote the ophthalmoscopically visible choroid and not in any exact anatomical sense. The "macular zone" extends from the fixation point to the $20^{\circ}$ circle concentric with the fixation point, the " mid-peripheral zone" from the $20^{\circ}$ to the $60^{\circ}$ circles, and the "extreme peripheral zone" from the $60^{\circ}$ circle to the region of the anatomical equator; Hepburn regarded each zone as a separate entity with only a few intervening capillary anastomoses. His conclusions, however, are not based on sound principles as has been shown above.

(3) There is doubt regarding the validity of the hypothesis that the periphery of the choroid is a distinct zone isolated from the rest of the choroid.

Indeed, the idea would appear untenable, since direct anastomoses have been shown to link the posterior and recurrent choroidal circulations in man at the equator, and since after optico-ciliary resection many areas of the choroid behind the equator may be spared (Komoto, 1915; Cogan, 1950), particularly if the anterior ciliary arteries are left intact (Berlin, 1871; Studer, 1906; Birch-Hirschfeld, 1910). Nor is it necessary to endow the choroidal periphery with any peculiar functional ability in order to explain the fact that after blockage of the central retinal artery the whole thickness of the retina in the extreme periphery may remain viable, whereas elsewhere the inner part of the retina becomes degenerate. It has been shown by Michaelson (1950) that the choroid in general is able to supply nourishment to the retina to a depth of about 130 microns. This thickness covers the pigment layer, the layer of rods and cones, and the outer nuclear and outer plexiform layers over the whole of the retina, except in the extreme periphery and at the macula where the entire retina narrows to about that thickness. It is not surprising, therefore, that the retina in the extreme periphery can be nourished exclusively by the choroid, and it is understandable that part of the transudate from the anterior part of the choroid may filter through the attenuated peripheral retina to contribute to the formation of aqueous humour; but these unusual features appear to be attributable to peculiarities of retinal rather than of choroidal structure. This is in keeping with the failure to find any specific features in the peripheral part of the chorio-capillaris in an examination of casts of the choroid prepared by the intravascular injection of Neoprene. It is true that the capillaries of the choroid are not uniform in calibre throughout the chorio-capillaris; posteriorly they are smaller with a closer capillary network, and anteriorly they are wider with a more open network, but this variation in calibre comes about gradually as one passes from the central to the peripheral parts of the choroid, and there is no sharp distinction between individual areas (Fig. 8, opposite). 


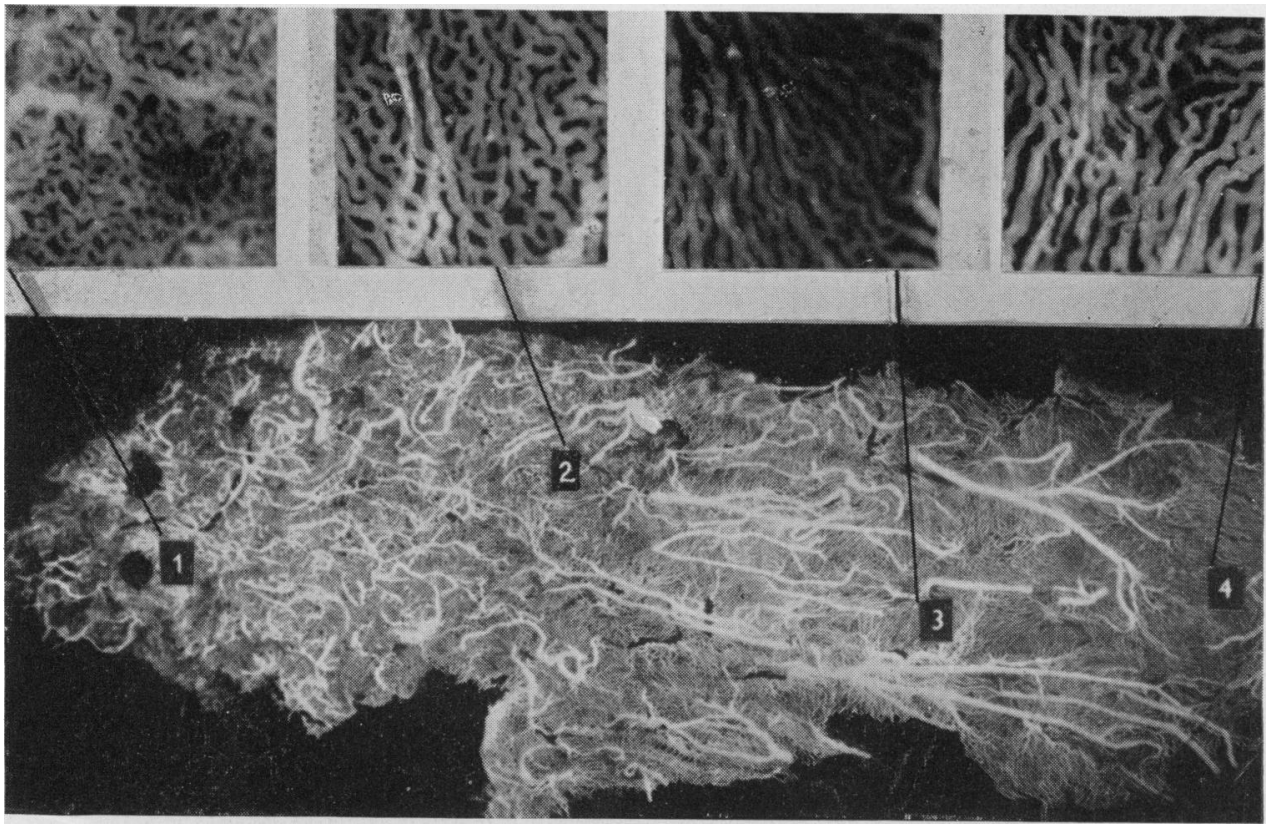

FIG. 8.-Neoprene cast of chorio-capillaris shcwing increase in calibre of capillaries and opening up of intercapillary spaces in passing from posterior to anterior parts of choroid, that is, from areas marked 1 to 4 . (Dissected specimen.) $\times 5$; insets $\times 24$. (Wybar, 1954.)

(4) There is doubt regarding the validity of the hypothesis that the choroid underlying the macula has distinctive features, at least as far as its capillary layer is concerned.

An examination of casts of the choroid injected with Neorrene did not reveal any structural differences between the chorio-capillaris underlying the macula and any other part of the chorio-capillaris situated an equivalent distance from the optic disc. There is, of course, a marked difference between scattered areas of the chorio-capillaris, in view of the gradual change in the calibre of the capillaries in passing from the central to the peripheral parts of the choroid; but it would appear that the density and calibre of the capillaries are purely expressions of the distance of the capillaries from the optic disc without any reference to particular areas. Vilstrup (1952), in an examination of casts of the chorio-capillaris in the rabbit prepared by intravascular injection of gelatin and of a plastic substance, did not detect any peculiar features of the chorio-capillaris underlying the macula, and Mörike (1949) found a uniform thickness of the chorio-capillaris throughout the choroid.

The submacular part of the choroid has a certain distinction, however, owing to the greater aggregation of arterial branches from the posterior ciliary arteries which lie within its area (Fig. 9, overleaf); in Neoprene casts this feature is only apparent after removal of the extensive network of choroidal veins which sweep round in the posterior part of the globe before passing to one of the main vortex veins (Fig. 10, overleaf). 


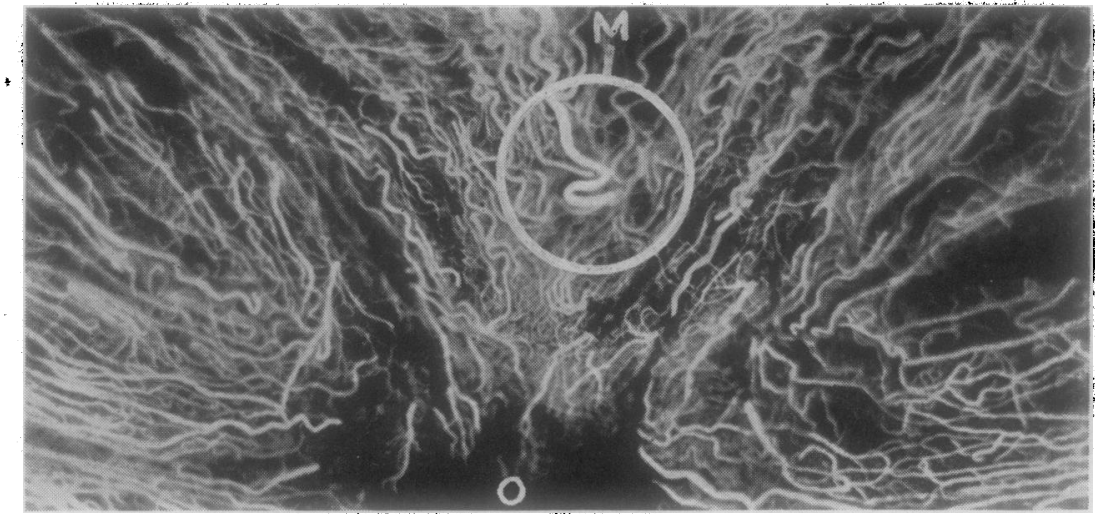

FIG. 9.-Neoprene cast of part of choroid surrounding optic disc (O), showing a greater number of arterial branches in submacular area $(M)$. (Dissected specimen in which majority of choroidal veins have been removed). $\times 6$.

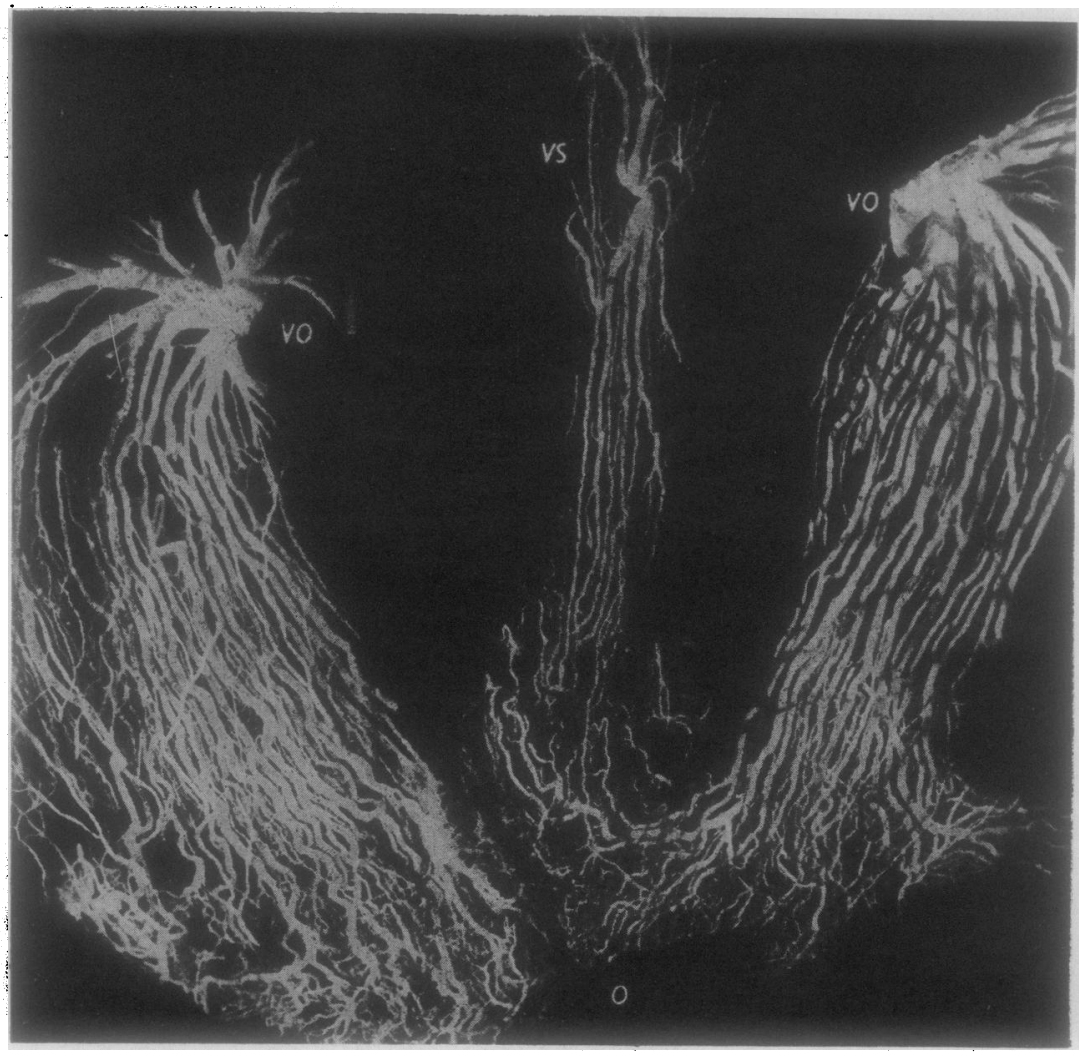

FIG. 10.-Neoprene cast of choroidal veins showing two main vortex vein systems (V.O.) and one subsidiary vortex vein system (V.S.). Note sweeping round of certain venules in peripapillary region $(O)$ as they run to the vortex veins. (Dissected specimen.) $\times 6$. (Wybar, 1054). 
It is interesting to speculate whether the increased arterial network of the submacular choroid is due merely to the fact that a greater number of posterior ciliary arteries perforate the globe in the neighbourhood of the posterior pole, or whether it arises as a physiological response to the higher metabolic demands of the specialized macula as postulated by Nettleship (1903). The latter view is supported by Mörike (1949), who found a well-marked thickening of the choroid under each macula in the bifoveal eye of the sea-swallow, despite the remoteness of either macula from the site of perforation of the ciliary arteries. On the other hand, from an anatomical point of view as has been mentioned above, the attenuation of the macular part of the retina is such that it may be nourished exclusively by the normal choroid, and in any event it is unlikely that a mere increase in the number of arteries affects the nutritive function of an area of choroid in the absence of specialized structural changes in the associated part of the chorio-capillaris.

(5) There is doubt regarding the validity of the hypothesis that the peripapillary region of the choroid is supplied by an exclusive system of arteries divorced from the rest of the choroid.

It is certainly true that within this area there are to be found many fine arterial branches which arise directly from the arterial circle of Zinn, as it lies within the sclera encircling the optic nerve, but many other fine arterial branches are derived from the short posterior ciliary arteries as they perforate the globe, and these cannot be considered to form an isolated part of the choroidal circulation. Furthermore, the capillaries in the peripapillary region are directly continuous with the main mass of the chorio-capillaris, so that there is no isolation of the peripapillary choroidal capillaries.

There does not appear, therefore, to be any clear-cut anatomical evidence to support the view that the selective localization of choroidal disease is attributable either to the involvement of isolated ciliary arteries or to peculiarities in the vascular architecture of certain parts of the choroid, and yet it is an undoubted feature of choroidal disease that the lesions tend to remain circumscribed. An area of choroid ceases to function only when the integrity of its capillaries is affected, and it has been suggested by Ashton (1953) that capillary damage may be brought about primarily by an increase in the normal tissue pressure within a localized region of the choroid. A likely cause for such an increase in pressure is a hæmorrhagic or inflammatory extravasation within the choroid, which exerts a marked effect on the choroidal vascular bed owing to the limitation of expansion imposed on the choroid by the normal force of the intraocular pressure acting against the unyielding sclera. The capillaries, by virtue of their delicate structure, are more readily affected than the other choroidal vessels by such a compressive force. This hypothesis provides a possible explanation for the isolated circular patches of choroidal capillary degeneration which occur in certain inflammatory and degenerative conditions, but it does not explain why such exudative or hæmorrhagic tendencies should so often occur in particular regions. 


\section{Summary}

(1) Casts of the choroidal vessels were prepared by the intravascular injection of Neoprene latex, and, owing to the cohesive and elastic properties of this substance, individual vessels and isolated capillary areas were able to be dissected from the main vascular mass and examined in detail.

(2) The short posterior ciliary arteries are segmentally arranged, and each branch supplies a localized zone of the choroid with an arteriolar-capillary network, but there is no anatomical evidence for the concept that these arteries are true end-arteries.

(3) The meeting place at the equator of the posterior choroidal circulation (short posterior ciliary arteries) and anterior choroidal circulation (recurrent arteries from the major arterial circle of the iris, from the long posterior ciliary arteries, and from the anterior ciliary arteries) may be marked by an intervening capillary network, but many of the vessels in the anastomosing circulations are in direct continuity with one another. This is contrary to the view that the equator is a zone of defective choroidal vascularization, and also to the view that the anterior part of the choroid is isolated from the rest of the structure.

(4) The size and density of the vessels in the chorio-capillaris vary in different regions according to their distance from the optic disc, but there is no evidence for the view that the chorio-capillaris underlying the macula has distinctive anatomical features.

(5) There is no anatomical evidence to suggest that the selective localization of choroidal disease is purely an expression of anatomical peculiarities of certain vascular districts.

I should like to thank Dr. Norman Ashton for introducing me to the technique of Neoprene injection and for his encouragement and advice throughout this investigation. I am indebted to Messrs. G. Knight and A. McNeil and to the Medical Illustration Department at the Institute of Ophthalmology for their help in the preparation of the casts and photographs.

\section{REFERENCES}

Ashton, N. (1952). British Journal of Ophthalmology, 36, 465. (1953). Ibid., 37, 140.

Bartolozzi, R. (1952). Arch. Soc. oftal. hisp.-amer., 12, 692.

BERLIN, R. (1871). Klin. Mbl. Augenheilk., 9, 278.

BirCh-HirsChFELD, A. (1910). Z. Augenheilk., 24, 193.

BOURNe, M. C., CAMPBell, D. A., and TANSLEY, K. (1938). British Journal of Ophthalmology, 22, 613.

COATs, G. (1907). Trans. ophthal. Soc. U.K., 27, 135.

CogAN, D. G. (1950). Trans. Amer. Acad. Ophthal. Otolaryng., 54, 629.

Collins, E. T. (1919). Trans. ophthal. Soc. U.K., 39, 165.

DUKE-Elder, S. (1940). "Text-book of Ophthalmology", vol. 3, p. 2102. Kimpton, London. (1948). Trans. ophthal. Soc. U.K., 68, 413.

Gonin, J. (1903). Ann. oculist. (Paris), 129, 24.

GREEVES, R. A. (1912). Roy. Lond. ophthal. Hosp. Rep., 18, 301.

HANCOCK, W. I. (1908). Ibid., 17, 428.

HEPBURN, M. L. (1910). Ibid., 18,92. (1912). Trans. ophthal. Soc. U.K., 32, 361.

Комото, G. (1915). Ibid., 35, 295. 
LaUber, H. (1931). In "Graefe-Saemisch Handbuch der gesamten Augenheilkunde", Bd. 1, Abt. 2. Springer, Berlin.

(1936). In "Handbuch der microskopischen Anatomie des Menschen", ed. W. von Möllendorff, vol. 3, pt. 2. Springer, Berlin.

Leber, T. (1903). In "Graefe-Saemisch Handbuch der gesamten Augenheilkunde ", 2nd. ed., Bd. 2, Abt. 2. Springer, Berlin.

Marín-Amat, M. (1952). Amer.J. Ophthal., 35, 1700.

MiCHAELSON, I. C. (1950). " XVI Concilium ophthalmologicum 1950, Britannia ", vol. 1, p. 605.

MörIKE, K. D. (1949). Klin. Mbl. Augenheilk., 114, 308.

NeTtLeSHIP, E. (1903). Roy. Lond. ophthal. Hosp. Rep., 15, 189.

NicholLs, J. V. V. (1938). British Journal of Ophthalmology, 22, 672.

PARRY, H. B. (1953). Ibid., 37, 487.

SalzmanN, M. (1912). "The Anatomy and Histology of the Human Eyeball", p. 57. University of Chicago Press.

StUder, T. F. (1906). Arch. Ophthal. (Chicago), 35, 333.

VERHOEFF, F. H. (1931). Ibid., 5, 392.

VILSTRUP, G. (1952). "Studies on the Choroidal Circulation". Munksgaard, Copenhagen.

Wagenmann, A. (1890). v. Graefes Arch. Ophthal., 36, pt. 4, p. 1.

Wolff, E., and Penman, G. G. (1950). "XVI Concilium ophthalmologicum 1950, Britannia ", vol. 1, p. 625.

Wolfrum, M. (1908). v. Graefes Arch. Ophthal., 67, 307.

WYBAR, K. C. (1954). J. Anat. (Lond.), 88, 94. 